\title{
Das Lößprofil von Ahlshausen und weitere tiefe Quartäraufschlüsse entlang der Bundesbahn- Neubaustrecke bei Northeim, Südniedersachsen
}

\author{
Heinz Jordan \& Wilhelm Schwartad *) \\ Loess, quaternary, soil stratigraphy, subrosion, \\ Lower Saxony
}

\begin{abstract}
Kurzfassung: In der Subrosionssenke von Ahlshausen wurde eine $25 \mathrm{~m}$ mächtige Lößlehm-Fließerde-Folge mit bis zu 9 Parabraunerden ( $\mathrm{fB}_{\mathrm{t}^{-}}$und Bleichhorizonte) untersucht. Unter dem 3. $\mathrm{fB}_{\mathrm{t}}$ fanden sich Reste eines Glaziärvorkommens, das eine elsterzeitliche Inlandvereisung belegt. Ein mächtiger Beckenschluff bis -ton im Liegenden der Lößfolge gehört vermutlich bereits in die Matuyama-Periode. In den Bahnprofilen und in Bohrungen der begleitenden Geologischen Kartierung wurden Kiesvorkommen mehrerer prä-holsteinzeitlicher Rhumeläufe nachgewiesen. Ihre Verstellung um viele Zehnermeter wird auf Hebung des Ahlshausener Gewölbes (über einem ZechsteinSalzkissen) zurückgeführt, unterbrochen durch lokale subrosive Absenkung.
\end{abstract}

\section{[The loess-profile of Ahlshausen and other deep quaternary exposures along the new railway at Northeim in the southern of Lower Saxony]}

\begin{abstract}
A 25 m-thick loess solifluction sequence including up to nine fossil soils (bleached layers and B horizons) was investigated in the subrosion basin of Ahlshausen which is cut by the German Federal Railways' new Hannover-Würzburg line. Pebbles of nordic (Scandinavian) provenance, incorporated in the loess sheet below the third fossil B horizon provide good evidence that the Elsterian inland ice-sheet reached the Ahlshausen area. A lacustrine silty clay sequence below the loess solifluction sequence probably belongs to the Matuyama magnetic period.

Gravel layers documenting several ancient courses of the Rhume river, of pre-Holsteinian age, were found in the railway cuttings and in drillholes during geological mapping of the area by the Lower Saxony Geological Survey. Vertical displacement of the Rhume sediments of the order of several tens of metres is related to young uplift of the Ahlshausen salt dome and to local subsidence by salt subrosion.
\end{abstract}

\footnotetext{
*) Anschriften der Verfasser: Dir. u. Prof. Dr. H. Jordan, Niedersächsisches Landesamt für Bodenforschung, Stilleweg 2, 3000 Hannover 51; W. SchWARTAu, Sophienstraße 19, 37574 Einbeck.
}

\section{Einleitung}

Die Bundesbahn-Neubaustrecke hat durch zahlreiche Untersuchungsbohrungen, Geländeeinschnitte und Tunnel für die Quartärgeologie des Leineberglandes einen enormen Kenntniszuwachs gebracht. Die baugeologische Betreuung in Südniedersachsen lag in Händen des Niedersächsischen Landesamtes für Bodenforschung (NLfB), Unterabteilung Ingenieurgeologie, unter Leitung von Prof. Dr. Drescher. Die Unterabteilung Geologische Kartierung hat gleichzeitig die Blätter der Geologischen Karte von Niedersachsen 1:25 000 neu bearbeitet und herausgegeben, deren Gebiet die Neubaustrecke durchquert (Blätter 4125 Einbeck, 4225 Northeim West, 4325 Nörten-Hardenberg).

Tabelle 1: Abschnitte der Bundesbahn-Neubaustrecke mit Aufschlüssen in alt- und mittelquartären Sedimenten, zwischen Northeim und Kreiensen (Lage s. Abb. 9).

\section{Baulos Stationen $(\mathrm{km})$}

A Dammstrecke bei

Edesheim

$$
217,310 \quad 74,5-76,8
$$

(Bohrungen, Abb. 7)

B Einschnitt bei Hohnstedt 217

$73-73,2$

C Kriebergtunnel, nördlicher Voreinschnitt (Tunnel in offener Bauweise) 284

D Einschnitt nördlich Ahlshausen $68,9(68,6-69,45)$

E Aue-Talbrücke 637

$64,25-65,5$

Im folgenden soll über Profile berichtet werden, in denen mittel- und altquartäre Schichten aufgeschlossen waren (Tab. 1). Ein Schlüsselprofil ist der Einschnitt bei Ahlshausen (C und D - Abb. 1), in dem W. SCHWARTAu eine $20 \mathrm{~m}$ mächtige Lößfolge mit bis zu neun Paläoböden aufnahm und dabei das bisher einzige nordische Glaziärvorkommen im Leinebergland südlich der Saaleeisgrenze fand (Kap. 2). H. JORDAN untersuchte in den Bahnaufschlüssen und 


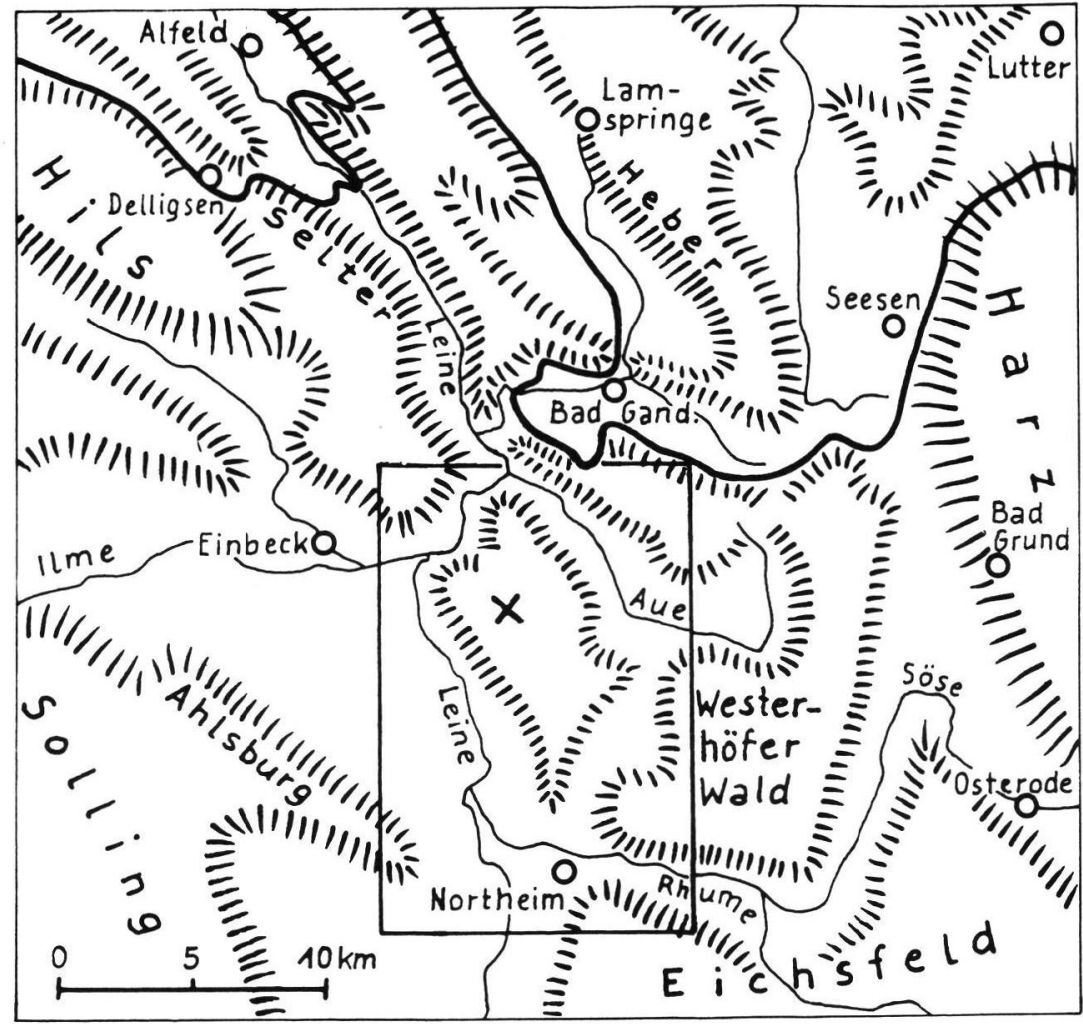

Abb. 1: Lage des Bahneinschnitts Alshausen (Kreuz) und maximale Ausdehnung des saalezeitlichen Inlandeises (starke Linie) im Leinebergland. - Rahmen = Ausschnitt der Abb. 9.

in Bohrungen der Geologischen Kartierung die Flußablagerungen einer Alt-Rhume (Kap. 3).

\section{Lößprofil von Ahlshausen}

Im Scheitel des Buntsandsteingewölbes von Ahlshausen ist ein $500 \mathrm{~m}$ breiter Graben eingebrochen. Bruchtektonisch angelegt, wurde er durch Subrosion beständig vertieft, so daß quartäre Sedimente sich in einer Mächtigkeit bis zu $50 \mathrm{~m}$ in einer Schutzsituation anreichern konnten. Die Schichtfolge wurde in den Einschnitten bei Ahlshausen (C und D der Tab. 1) nahezu $25 \mathrm{~m}$ tief freigelegt und bis $45 \mathrm{~m}$ tief abgebohrt. Im folgenden werden zwei Lößprofile beschrieben, die etwa $1000 \mathrm{~m}$ voneinander entfernt liegen. Die Schichten beider Profile lassen sich in nahezu horizontaler Lage nach den Seiten hin ohne erkennbare tektonische oder subrosive Störungen verfolgen. Sie sind aber verschiedentlich durch erosive Prozesse gestört; dadurch wird eine Verknüpfung der Schichten über größere Entfernung unsicher.

\subsection{Profil}

\section{Ahlshausen 1}

Das Profil liegt etwa in der Mitte des Abschnitts C der Tab. 1 , bei R: $3566820, \mathrm{H}$ : 5740 430. In Abb. 2 ist die Schichtfolge schematisch dargestellt. Im folgenden wird die Westseite des Bahneinschnitts beschrieben.

Das Profil ist in seinem oberen Teil in einer schmalen Kuppe erhalten, die durch seitliche Erosion vor allem während des letzten Glazials entstanden ist. Die bis zu $8 \mathrm{~m}$ tiefen Dellen sind während des Jungwürm und Holozän wieder verfüllt, so daß die Kuppe morphologisch nicht mehr erkennbar ist. Die Sedimente des Würm ${ }^{1)}$ überziehen auch die Kuppe in einer Mächtigkeit von $2 \mathrm{~m}$. Sie tragen an ihrer Oberseite den Rest der holozänen Parabraunerde über dem einzigen kalkhaltigen Löß des ganzen Profils und darunter offenbar umgelagerte Reste des Lohner Bodens, der in seitwärts gelegenen mächtigeren Würmprofilen rasch in die Naßfazies des Hattorfer Bodens übergeht.

Darunter folgt eine Serie fossiler Tonanreicherungshorizonte $\left(\mathrm{fB}_{\mathrm{t}}\right)$, fast jeder von einem geringmächtigen Bleichhorizont überdeckt. In diesem steckt weitgehend umgelagertes $\mathrm{A}_{1}$-Horizont-Material. Die $\mathrm{fB}_{\mathrm{t}}$-Horizonte lassen sich über den Einschnitt hinweg konnektieren und sind auf der West- wie Ostseite einheitlich numeriert (Abb. 2-4).

Der oberste Bleichhorizont weist - wie mehr oder weniger auch die älteren Bleichhorizonte - an seiner Basis eine Anreicherung von Mangan-Eisenhydroxid-Konkretionen als Kennzeichen von Pseudovergleyung auf, sowie geringe Gehalte von Holzkohle. Da der Horizont durch kleine Eiskeilpseudomor-

1) "Würm" wird in der Paläopedologie traditionsgemäß auch in Norddeutschland synonym für "Weichsel"(-Kaltzeit) verwendet. 


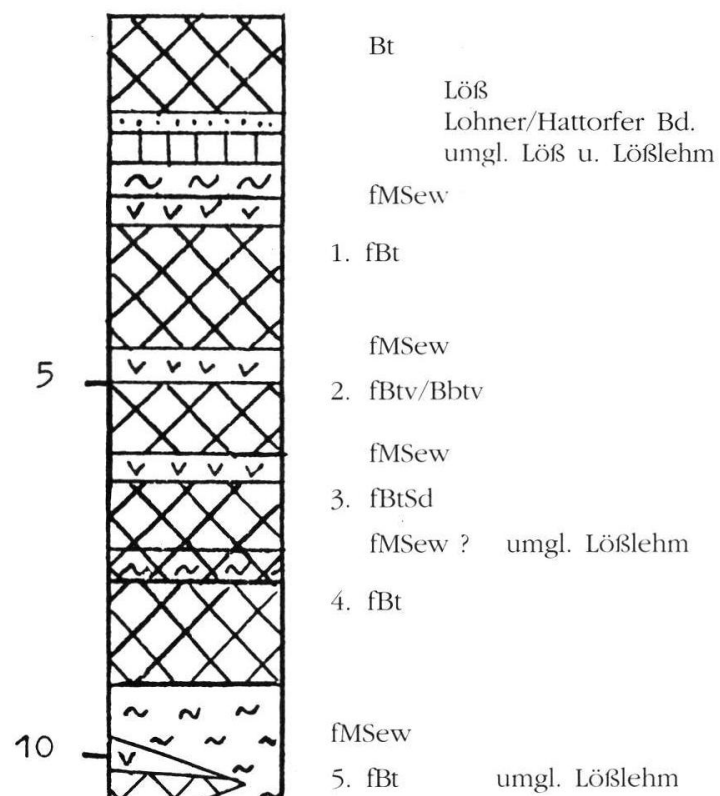

15

20

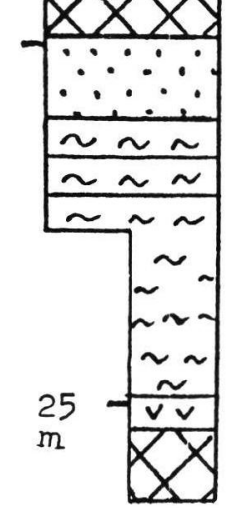

8. $\mathrm{fBt} \mathrm{Sd}$

Lößlehm

Sandsteinschutt umgl. Beckenton

umgl. verlehmter Rotsand mit Sandsteinen

fMSew

9. $\mathrm{fBt}$

umgl. Rotsand u. Lößlehm phosen mit dem liegenden $1 . \mathrm{fB}_{\mathrm{t}}$ verzahnt ist, kann er genetisch nicht diesem als Oberboden zugeordnet werden. Der 1 . $\mathrm{fB}_{\mathrm{t}}$ ist, seitwärts nur unterbrochen von Dellen, durchgehend bis zum Profil Ahlshausen 2 (Kap. 2.2) zu verfolgen. Als beständiger, mächtiger und sehr ausgeprägter Horizont ist er dem Eem zuzuordnen. Mit seiner kräftigen Tonanreicherung, mit deutlichen Tonbelegen auf den Aggregatoberflächen und brauner Farbe mit nur sehr geringer Marmorierung ist er Teil einer Parabraunerde. Wegen der Frostkeile muß die Grenze Eem-/Würm an die Oberfläche der gekappten Parabraunerde gelegt werden. Das Bleichhorizontmaterial mit deutlich laminar-solifluidaler Struktur entstammt aber vermutlich weitgehend dem abgetragenen A-Horizont der Parabraunerde und ist daher ein Koluvium des beginnenden Würm.

Die nach unten abnehmende Tonanreicherung des Eembodens geht ohne deutliche Grenze in die nur schwach ausgeprägte Lamellenfleckenzone über, die aber in einer tiefer reichenden Eiskeilpseudomorphose mit besserer Perkolation kräftig ausgebildet ist. Die Lamellierung hat auch schwach auf den oberen Teil des liegenden 2. Bleichhorizontes übergegriffen. Da aber dessen unterer Teil keine makroskopisch erkennbare Tonverlagerung besitzt, kann die Lamellierung des unter dem 2. Bleichhorizont liegenden Restes einer mäßig pseudovergleyten Parabraunerde nicht vom hangenden Eemboden ausgegangen sein, sondern ist autochthoner Bestandteil des $2 . \mathrm{fB}_{\mathrm{t}}$.

Ein 3. Bleichhorizont mit zahlreichen Konkretionen liegt dem 3. $\mathrm{fB}_{\mathrm{t}}$ auf, bei dem eine Lamellierung wegen starker Pseudogleybahnen nur undeutlich erkennbar ist. Die unter dem Bleichhorizont umgebogenen Pseudogleybahnen kennzeichnen eine solifluidale Beanspruchung nach der Abtragung des Oberbodens der Parabraunerde. Eine $85 \mathrm{~cm}$ mächtige Umlagerungszone enthält neben gelbem und bräunlichem Lößlehm ohne Tonbelege kräftige graue und naßbodenartige Bänder, wie sie auch aus dem beginnenden Jungwürm bekannt geworden sind.

Wenige zwischengeschaltete Streifen mit Bleichhorizontmaterial dürften dem ursprünglich über dem 4. $\mathrm{fB}_{\mathrm{t}}$ gelegenen Bleichhorizont angehört haben. Tonbelege in diesem $4 . \mathrm{fB}_{\mathrm{t}}$ sind zwar nur schwach ausgeprägt, so daß er lediglich der basale Rest dieses $\mathrm{B}_{\mathrm{t}}$ ohne Lamellierung sein dürfte. Fahnenförmig gebogene Pseudogleybahnen unter der Umlagerungszone belegen aber seine Eigenständigkeit gegenüber dem 3. $\mathrm{fB}_{\mathrm{t}}$. Der 4. $\mathrm{fB}_{\mathrm{t}}$ geht zum Liegenden über in eine Umlagerungzone von grauem, violettem, stroh- oder matt-gelbem Lößlehm in raschem Wechsel. Das Material dürfte z. T. kaltzeitlichen Naßböden entstammen, ohne daß eine Gliederung möglich ist.
Abb. 2: Profil Ahlshausen 1, schematisch. - Punkte = Lößlehm, Häkchen = Bleichzone, Karo = verbraunter Tonanreicherungshorizont, Tilden $=$ Schluff bis Sand, solifluidal umgelagert, Dreiecke $=($ zum Teil mit Steinen $)$. 
Zonale Rostflecken und Rostbänder, feine Pantherung und Eisenhydroxid-verfestigte Wurzelbahnen gehören einer Überprägung nach der Ablagerung an. In dieser Umlagerungszone verschwindet ein - $15 \mathrm{~m}$ weiter südlich erhaltener $-5 . \mathrm{fB}_{\mathrm{t}}$, der hier also der Erosion zum Opfer gefallen ist.

Darunter liegt ein Bleichhorizont, der zusammen mit dem basalen Teil der hangenden Umlagerungszone und dem oberen Teil des liegenden $6 . \mathrm{fB}_{\mathrm{t}}$ sekundär so naßgebleicht wurde, daß der Eindruck einer einheitlichen $80 \mathrm{~cm}$ mächtigen Bleichzone erweckt wird. Die Bleichung ist so kräftig, daß ein Horizont kleiner solifluidal geneigter Eiskeilpseudomorphosen, mit denen Bleichhorizont und liegender $6 . \mathrm{fB}_{\mathrm{t}}$ verzahnt sind, kaum zu erkennen ist.

Zwischen 6. und 7. $\mathrm{fB}_{\mathrm{t}}$ fehlt ein Bleichhorizont. Die Grenzfläche ist statt dessen markiert durch eine schmale Umlagerungszone, an der kleine Eiskeilpseudomorphosen ansetzen. Wenn auch der $7 . \mathrm{fB}_{t}$ vom 6. $\mathrm{fB}_{\mathrm{t}}$ etwas überprägt sein dürfte, wie die sehr deutliche Lamellierung der Eiskeilpseudomorphosen beweist, so ist seine Selbständigkeit doch dadurch gesichert, daß in jedem der zwei Böden eine kompakte Tonanreicherung nach unten in eine deutliche Lamellierung übergeht. Dem 7. $\mathrm{fB}_{t}$ mit markanter Verbraunungsuntergrenze ist dichter Lößlehm unterlagert, der zunehmend in durch Vergleyung blau gefärbten Lößlehm übergeht.
Darunter schaltet sich in die Löß-Böden-Serie eine Lage roten Buntsandsteinschutts aus Ton bis Schluff, Sand und Steinen bis $30 \mathrm{~cm}$ Durchmesser ein, durchsetzt mit blaugrauem Lößlehm.

Unter einem schwach ausgeprägten matt gelbgrauen Bleichhorizont mit sehr wenig Konkretionen folgt der stark pseudovergleyte $8 . \mathrm{fB}_{\mathrm{t}}$ mit nahezu horizontalen Pseudogleybahnen als Zeichen einer kräftigen solifluidalen Überformung. Offenbar wegen der starken Überformung konnten die für die Parabraunerde typischen Tonbelege nur seitwärts des Profils gefunden werden, wo Feuchtüberprägung und wohl auch die Durchmischung geringer waren. Darunter folgt gelber, grau gepantherter und schließlich blaugrauer Lößlehm. In einer Bohrung fand sich darunter ein weiterer Bleichhorizont mit dem oberen Teil eines 9. $\mathrm{fB}_{\mathrm{t}}$.

Insgesamt zeigt das Profil nach unten Spuren zunehmender Vernässung und solifluidaler Überformung. Über die tieferen, nicht mehr aufgeschlossenen Schichten gibt eine Trassenbohrung Auskunft (I 800 der GK 25, Blatt 4225 Northeim West), die ungefähr am linken Rand des Profils Abb. 3/4 steht. Sie durchsank bis $33 \mathrm{~m}$, also bis $7 \mathrm{~m}$ unterhalb des 9 . $\mathrm{fB}_{\mathrm{t}}$, eine Wechselfolge von sandigem Schluff und schluffigem Ton mit Lagen von Buntsandstein-Fließerde, darunter bis $50 \mathrm{~m}$ grauen schluffigen Ton (Beckenton). Bei der Endteufe von $51 \mathrm{~m}$ kündigt rötlicher Schluff die Basis der Beckensedimente an, in ca. $55 \mathrm{~m}$ Tiefe ist

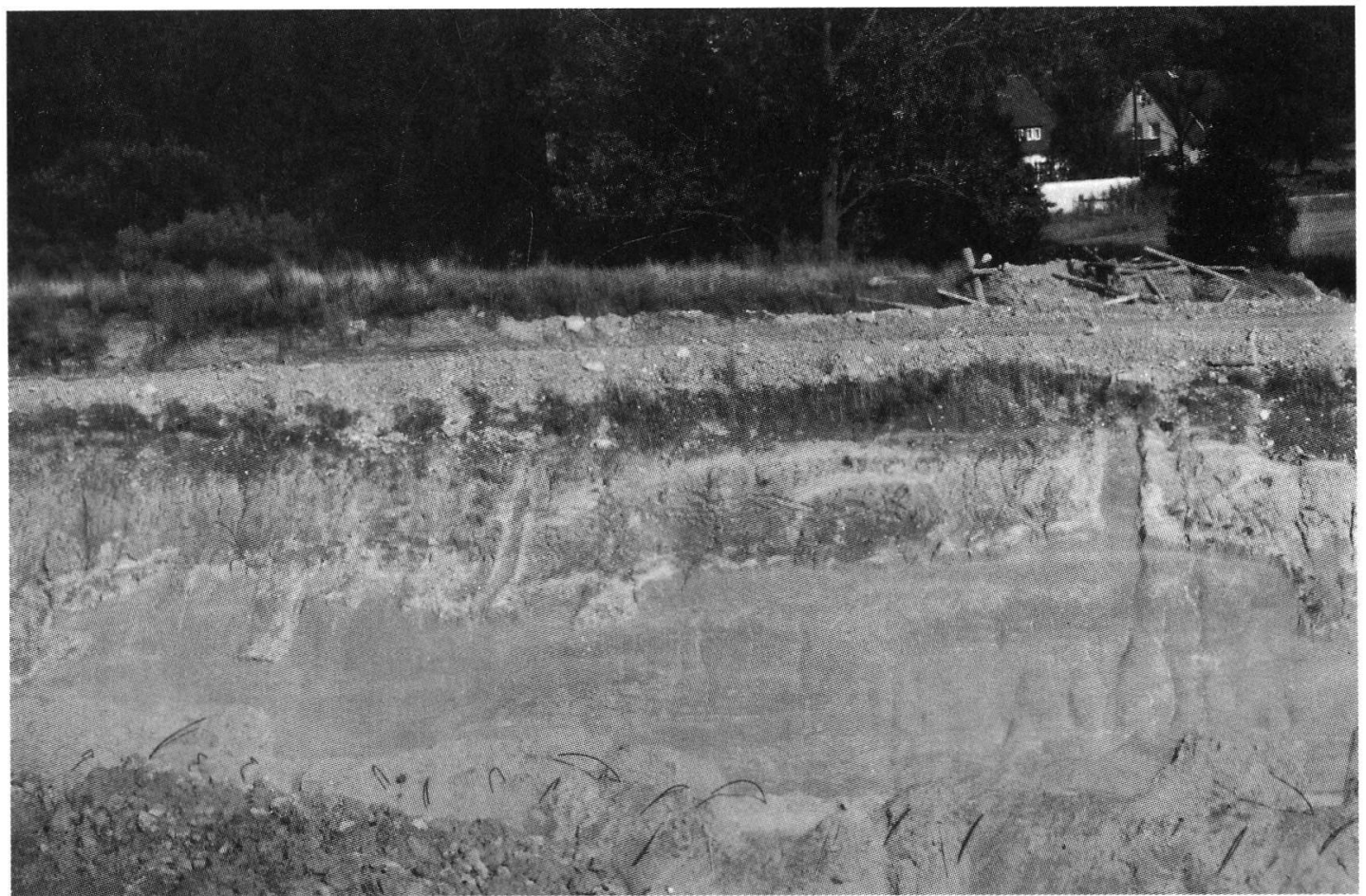

Abb. 3: Profil Ahlshausen 1 
N

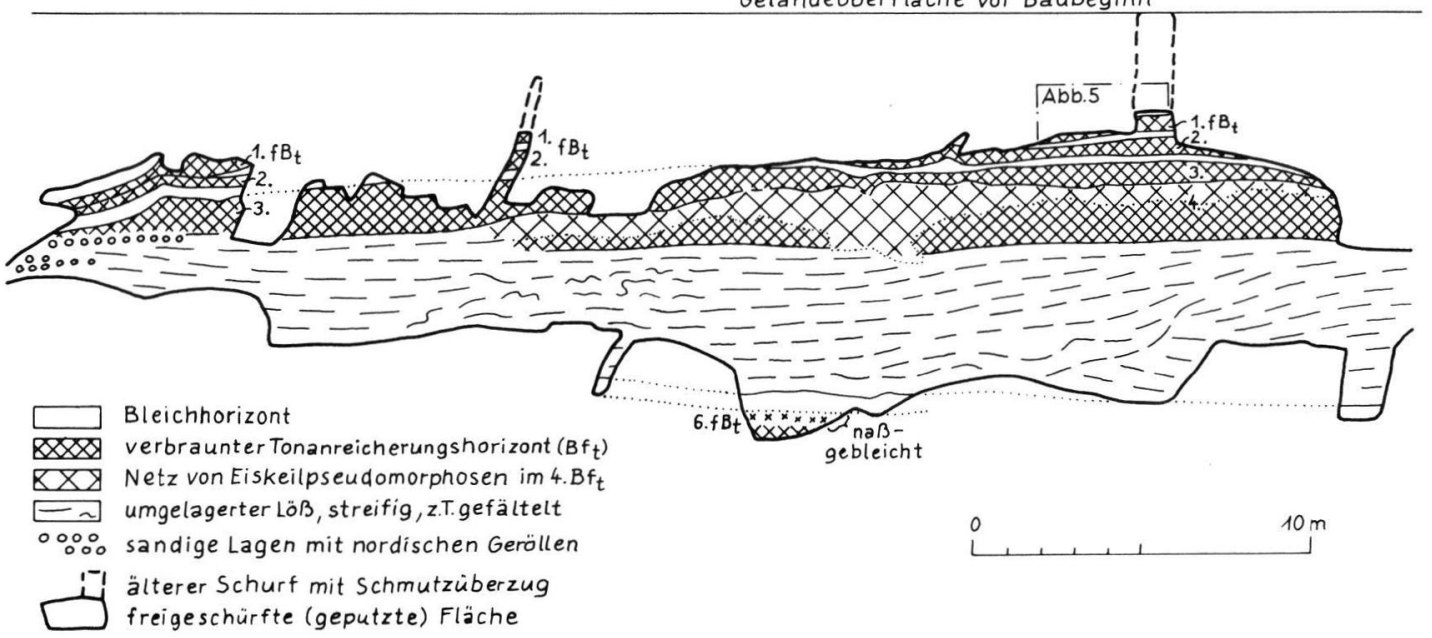

Alb. 4: Profil Ahlshausen 1 nach dem Foto Abb. 3

nach flachseismischer Erkundung anstehender Buntsandstein zu erwarten.

\section{Ostseite des Bahneinschnittes}

Die beiden Dellen, welche die nur $20 \mathrm{~m}$ breite Kuppe des Westprofils mit dem Eemboden herausgearbeitet haben, laufen nach Osten auseinander, so daß die Kuppe an der EinschnittOstseite etwa $100 \mathrm{~m}$ Breite erreicht. Im rechten Teil der Abb. 3, die einen $40 \mathrm{~m}$ breiten Abschnitt dieser Kuppe zeigt, sind der 1. und 2. $\mathrm{fB}_{\mathrm{t}}$ mit ihren Bleichhorizonten stark ausgedünnt mit nahezu waagerecht verlagerten Pseudogleybahnen. Solche Bahnen, die aus dem 3. Bleichhorizont, z. T. auch aus dem $2 . \mathrm{fB}_{\mathrm{t}}$ in den $3 . \mathrm{fB}_{\mathrm{t}}$ ziehen, belegen, daß Bodenbildung und Pseudovergleyung mindestens teilweise aufeinander folgende Prozesse sein können, wie auch die kräftige Konkretionsanreicherung $\mathrm{im} 3 . \mathrm{fB}_{\mathrm{t}}$ sekundär sein kann.

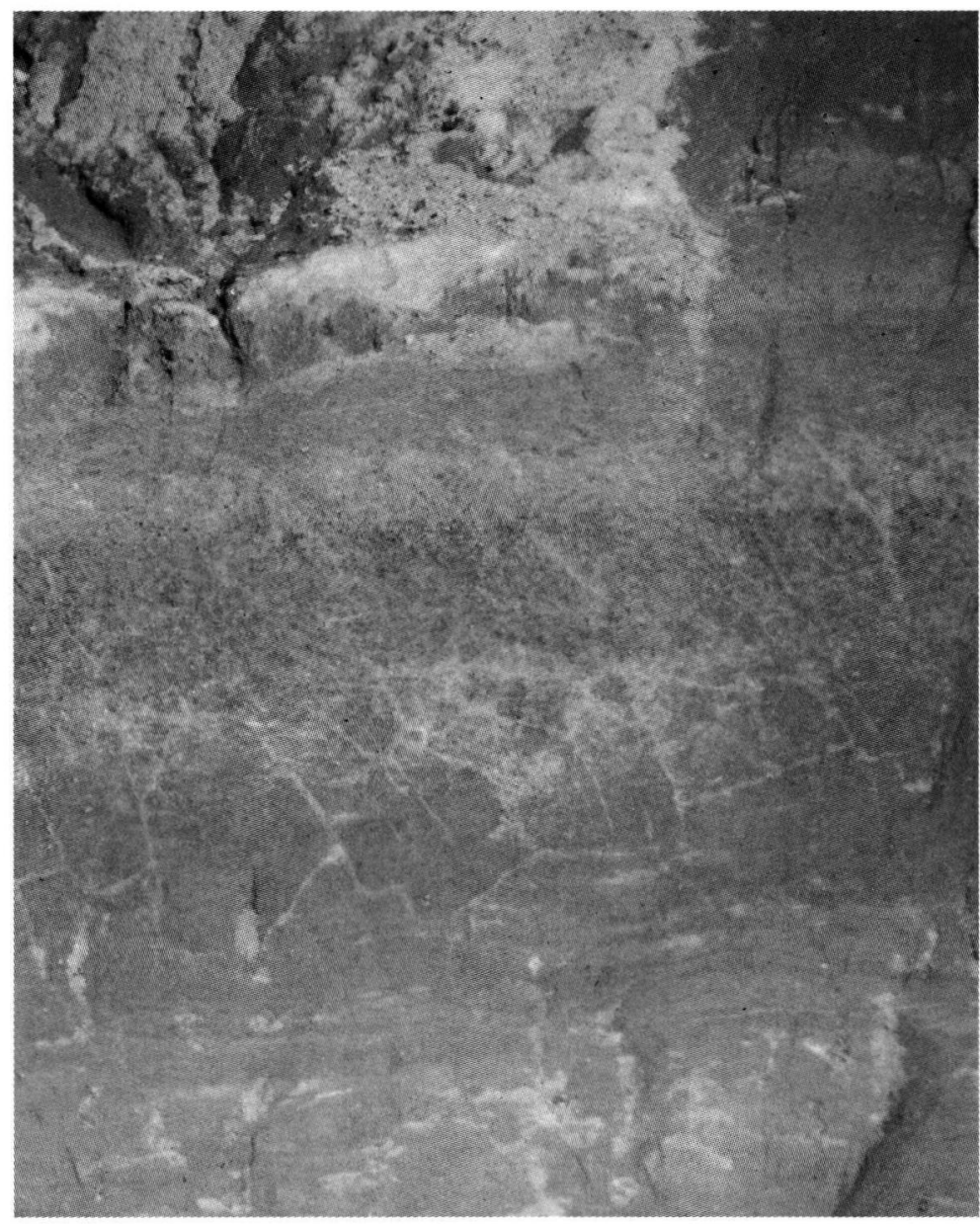

Abb. 5: Profil Ahlshausen 1, Ausschnitt (Lage s. Abb. 4). 
Der stark mit Ton angereicherte, umgelagerte und rasch austrocknende obere Teil des 4 . $\mathrm{fB}_{\mathrm{t}}$ trägt ein Netz von schmalen Pseudogleybahnen, die offenbar schmale Eiskeilpseudomorphosen nachzeichnen und in den liegenden Teil des Bodens übergreifen (Abb. 4). Toninfiltrationsbänder an dessen Basis belegen den autochthonen Charakter des liegenden Teils des $4 . \mathrm{fB}_{\mathrm{t}}$. Eine Eiskeilpseudomorphose vor allem mit Material des oberen Teils dieses Bodens reicht bis in die darunter folgende mächtige Zone mit laminar solifluidal verflossenem Lößlehm mit Resten aus mindestens drei Naßböden. Zusammen mit den darunter folgenden Bodenschichten entspricht im großen und ganzen das Profil der Ostseite dem der Westseite. Auf der Ostseite fehlt allerdings der 5. $\mathrm{fB}_{\mathrm{t}}$ ganz.

Nach Norden, in Richtung auf die begrenzende Delle (Abb. 3/4, linker Rand) sind 1. bis 3. $\mathrm{fB}_{\mathrm{t}}$ zunehmend laminar verflossen. Der 3. $\mathrm{fB}_{\mathrm{t}}$ ist durch Pseudogleybahnen, die in diesem Boden wurzeln, aber im Bild nach links umbiegen, überprägt. Dadurch wird der autochthone oder parautochthone Charakter des Pseudogleys - nicht unbedingt auch des Bodens - bezeugt. Der laminar verlagerte Lößlehm unter diesem Pseudogley enthält nahe der Delle einzelne mm- bis cm-starke sandig-grusige Lagen mit Geröllen nordischer Herkunft (Abb. 4, Tab. 2). Sie sind älter als der 3. $\mathrm{fB}_{\mathrm{t}}$. Die Gerölle müssen aus einem höher, $d$. h. westlich bis südlich gelegenen Glaziärvorkommen (Schmelzwasserkies, Moräne?) stammen und mit dem Lößlehm verlagert worden sein. Wegen der Verknüpfung der laminaren Strukturen mit dem Lößlehm, der den im linken Bilddrittel aussetzenden 4 . $\mathrm{fB}_{\mathrm{t}}$ trägt, könnten die Gerölle allerdings auch älter sein als der $4 . \mathrm{fB}_{\mathrm{t}}$.

Tabelle 2: Geröllbestand (Durchmesser $3 \mathrm{~mm}$ bis $5 \mathrm{~cm}$ und ein faustgroßer Granit) der sandig-grusigen Lagen im Profil Ahlshausen 1.

Geröll.

Anzahl

Nordisch:

Kristallin (Granit, Gneis, Amphibolit,

Glimmerschiefer)

Quarzit

Flint

Paläoziokum (vom Harz):

Grauwacke

Kieselschiefer

Quarzit, Kahlebergsandstein

Gangquarz

Mesozoikum:

Buntsandstein (überwiegend von Hängen um Ahlshausen)

Rogenstein (vom Raum Seesen oder weiter zahlreich,
nicht gesammelt nördlich)

Hilssandstein, Flammenmergel (von Norden)

Toneisenstein (Jura-Geodentrümmer von Norden)
Der Kristallin-Anteil enthielt nach K.-D. MEYER (NLfB Hannover) keine Leitgeschiebe, ist aber eindeutig skandinavischer Herkunft. Im paläozoischen Anteil fand sich der charakteristische Kahlebergsandstein vom Nordharz, dagegen kein Acker-BruchbergQuarzit oder Südharz-Porphyr. Das Glaziärvorkommen stammt also von einem den Nordharz streifenden Eisstrom.

\subsection{Profil Ahlshausen 2}

Es liegt $950 \mathrm{~m}$ nördlich vom Profil Ahlshausen 1 bei R: 3566 940, H: 5741370 (D der Abb. 9) und ist in Abb. 6 dargestellt.

In die Lößfolge mit sechs fossilen Böden schalten sich ab $4 \mathrm{~m}$ Tiefe sandig-kiesige Lagen ein, z. T. Buntsandstein-Fließerden, z. T. fluviatiler Kies mit gut gerundeten Buntsandstein- und paläozoischen Geröllen (Rhume-Oberterrasse, Kap. 3). Die Hauptmasse des fluviatilen Kieses liegt unter dem 3. $\mathrm{fB}_{t}$, z. T. einbezogen in dessen Tonanreicherungszone (A in Abb. 6). Doch finden sich Geröllagen, immer auch mit Paläozoikum-Anteil, bis hinab zu einem weißgrauen, stark kalkhaltigen Beckenschluff, der eine gut $20 \mathrm{~m}$ mächtige Folge feinkörniger Stillwassersedimente einleitet.

Meist stecken die fluviatilen Gerölle in umgelagertem Lößlehm und sind offenbar eingeschwemmt. Bei B (= Geröllanalyse 6 der Tab. 3) und C der Abb. 6 liegt wohl Rhume-Fluviatil in situ vor: toniger Schluff bis Kies mit hohem paläozoischem Geröllanteil.

\subsection{Zum Alter der Schichten}

Lößboden-Stratigraphie ist in vielen Gebieten erfolgreich angewendet worden. So ist mit ROHDENBURG \& MEYer (1966) der oberste $\mathrm{fB}_{\mathrm{t}}$-Horizont bei Ahlshausen - stets unter Würmlöß mit seinen charakteristischen Humuszonen anzutreffen - ins Eem zu stellen. Vermutlich sind die tieferen, durchgehend verbraunten $\mathrm{fB}_{\mathrm{t}}$-Horizonte Bildungen älterer Interglaziale, aber leider verbietet sich eine Datierung nach der Abzählmethode. Einmal können Bodenhorizonte erodiert sein (Abb. 2: $5 . \mathrm{fB}_{\mathrm{t}}$ ), zum andern können geringermächtige $\mathrm{Bt}-$ Horizonte auch in Interstadialen entstehen, oder zwei $\mathrm{B}_{\mathrm{t}}$-Horizonte in einem Interglazial (STREMme et al. 1991).

Wir wollen uns daher im folgenden soweit wie möglich auf geologische Befunde stützen, um zu einer Altersabschätzung zu kommen.

2 Der graue Ton bis Schluff, der im gesamten Ahlshausener Becken 20-30 m mächtig Löß und Fließerden unterlagert, war an zwei Stellen für eine pol- 


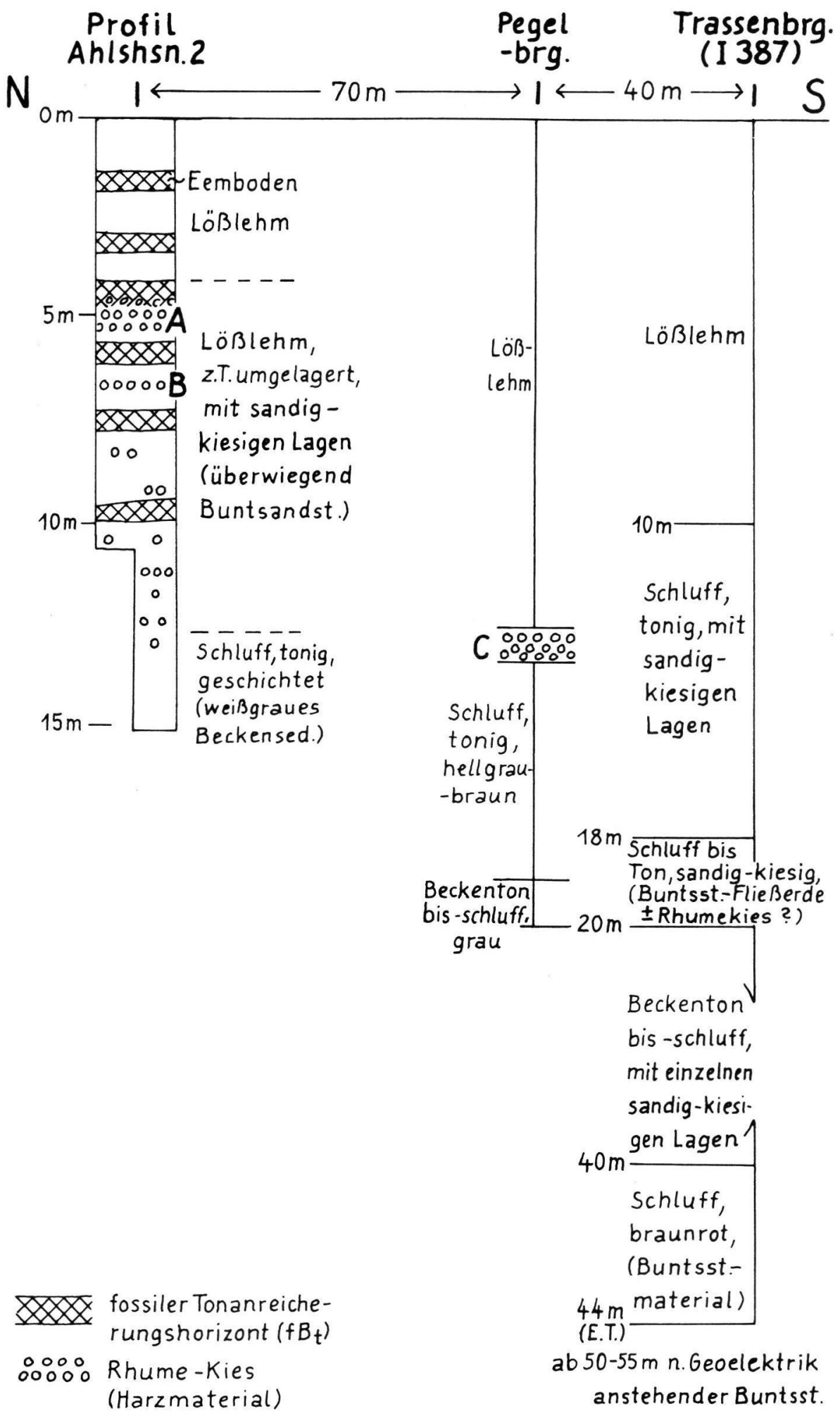


lenanalytische Untersuchung zugänglich. $100 \mathrm{~m}$ nördlich von Profil Ahlshausen 1 wurde grüngrauer feinsandiger Beckenton in der Trassensohle freigelegt, $3 \mathrm{~m}$ tief abgebohrt und von E. Grüger (Univ. Göttingen, freundl. schriftl. Mitt.) auf Pollen untersucht: Der Ton ist pollenfrei bis auf eine Lage, die neben umgelagerten Baumpollen ("allenfalls die Kiefer wuchs in der Nähe") überwiegend Seggen und Sphagnen führt, was für kaltzeitliche Ablagerung in einem See spricht.

Auch der weißgraue Schluff des Profils Ahlshausen 2 (Abb. 6) enthält nach H. MüLLER (ehemals BGR Hannover) neben mesozoischen Sporomorphen nur Kiefern- und Graspollen und ist somit eine kühl- bis kaltklimatische Ablagerung. Damit entfällt an beiden Stellen die Möglichkeit einer Alterseinstufung ( $\mathrm{Zu}-$ ordnung zu einer bekannten Warmzeit).

Nach einer paläomagnetischen Untersuchung des Profils Ahlshausen 1 durch Fromm (1988) liegt die Grenze Brunhes/Matuyama, die stratigraphisch ins Cromer-Glazial A gestellt wird, vermutlich in der Schwemmlöß-Rotsand-Folge in $17 \mathrm{~m}$ Teufe (vgl. Abb. 2). Allerdings gibt lediglich eine geringe Winkeldifferenz in der Inklination einen Hinweis auf ehemals inverse Magnetisierung, die durch chemische Überprägung ausgelöscht sein soll. Das Ergebnis ist immerhin ein Anhalt dafür, daß die Lößfolge oberhalb $17 \mathrm{~m}$ mit 2 bis 3 Böden den höheren $\mathrm{Ab}$ schnitt des Cromer-Komplexes vertreten könnte. Bleibt schließlich das Glaziärvorkommen im Profil Ahlshausen 1. Das niedersächsische Bergland ist in seinem Nordteil zweimal vom nordischen Inlandeis überfahren worden: in der Elster- und in der SaaleKaltzeit (Drenthe-Stadium). Eine noch ältere Vereisung läßt sich nirgendwo in Norddeutschland nachweisen. Nun liegt der Saale-Eisrand, durch Grundmoräne und Vorschütt-Kiessand gut belegt, viele km nördlich von Ahlshausen (Abb. 1); nämlich in den Talungen bei Alfeld-Freden, Bad Gandersheim und Seesen (Gk 25, Blätter 4025 Freden, 4125 Einbeck und 4127 Seesen).

Vom Elster-Glaziär ist so weit südlich fast nichts erhalten geblieben. Obwohl LütTıgs (1960) Datierungen elsterzeitlicher Kiese heute nicht mehr haltbar sind, gibt es Hinweise, daß das Elster-Inlandeis weiter nach Süden vordrang als das Saaleeis (GK 25, Blatt 4023 Eschershausen). Das Glaziärvorkommen von Ahlshausen muß, weil es außerhalb der Saaleeisverbreitung liegt, der elsterzeitlichen Inlandvereisung zugeordnet werden. Der Gletscher muß, um das $180 \mathrm{~m}$ hoch gelegene Ahlshausener Becken zu erreichen, in breiter Front die umrandenden Höhen überfahren haben und in den Talungen noch weiter nach Süden, sicher bis Einbeck und vielleicht bis Northeim vorgedrungen sein.
Die geologischen Befunde lassen also für das Profil Ahlshausen 1 folgende Alterseinstufung möglich erscheinen:

1. $\mathrm{fB}_{\mathrm{t}}$

Eem-Warmzeit

2. und 3. $\mathrm{fB}_{\mathrm{t}}$, bzw. 2. bis $4 . \mathrm{fB}_{\mathrm{t}}$ - Warmzeiten

zwischen

Eem und Elster-

Kaltzeit (einschließ-

lich des Holstein)

nordische Gerölle

- Elster-Kaltzeit

ältere $\mathrm{fB}_{\mathrm{t}}$

Ahlshausener Beckenton

- Cromer-Komplex

- prä-Cromer

\section{Rhume-Oberterrasse zwischen Northeim und Kreiensen}

\subsection{Vorkommen}

Im Profil Ahlshausen 2 (Kap. 2.2) und in den anderen Bahnaufschlüssen der Tab. 1 fanden sich $\mathrm{Ab}-$ lagerungen der Alt-Rhume, gekennzeichnet durch paläozoische Gerölle vom Harz, die bis dahin nur vom Dünetal bei Northeim bekannt waren (vON KOENEN 1894: 76, LüTTIG 1954).

A. Bohrungen bei Edesheim haben unter $50 \mathrm{~m}$ mächtigem Mittelterrassenkies der Rhume einen älteren Schluff bis Kies durchteuft, der völlig frei von Rhume-Material, d. h. von paläozoischen Geröllen ist (Abb. 7). Der Umschwung in der Geröllzusammensetzung konnte pollenanalytisch ins ausgehende Holstein eingestuft werden. Zu dieser Zeit muß die Rhume ihren alten Lauf im Dünetal verlassen und den heutigen Durchbruch zur Leine bei Northeim geschaffen haben.

B. Im Bahneinschnitt östlich Hohnstedt (an und nördlich der Straßenbrücke bei R: 3566 870, H: 5737 050) war ein $40 \mathrm{~m}$ mächtiges Schichtpaket von karbonatisch verkittetem Kiessand aufgeschlossen, unten mit $1 \mathrm{~m}$ dicken Einschaltungen aus rötlichem Sand und dunkelgrauem Schluff. Es fällt auf der gesamten Länge $(120 \mathrm{~m})$ einheitlich mit $20^{\circ}$ nach Südwesten ein. $80 \mathrm{~m}$ nördlich der Brücke taucht das Liegende, roter Keupermergelstein, in der Bahntrasse auf, bei rund $130 \mathrm{~m}$ über $\mathrm{NN}$.

Das Kiessandpaket liegt dort hoch über der LeineMittelterrasse, deren Oberfläche nur $125 \mathrm{~m}$ über NN erreicht. Es muß sich also um ein Oberterrassen-Vorkommen handeln. Dafür spricht auch die starke Verkippung infolge Subrosion, die bei der Mittelterrasse nicht oder mit minimaler Neigung beobachtet wurde (GK 25 Blatt 4225 Northeim West). Die Geröl- 
N

$\mathrm{s}$

1769

12

131770

14

15

S 27

T 14

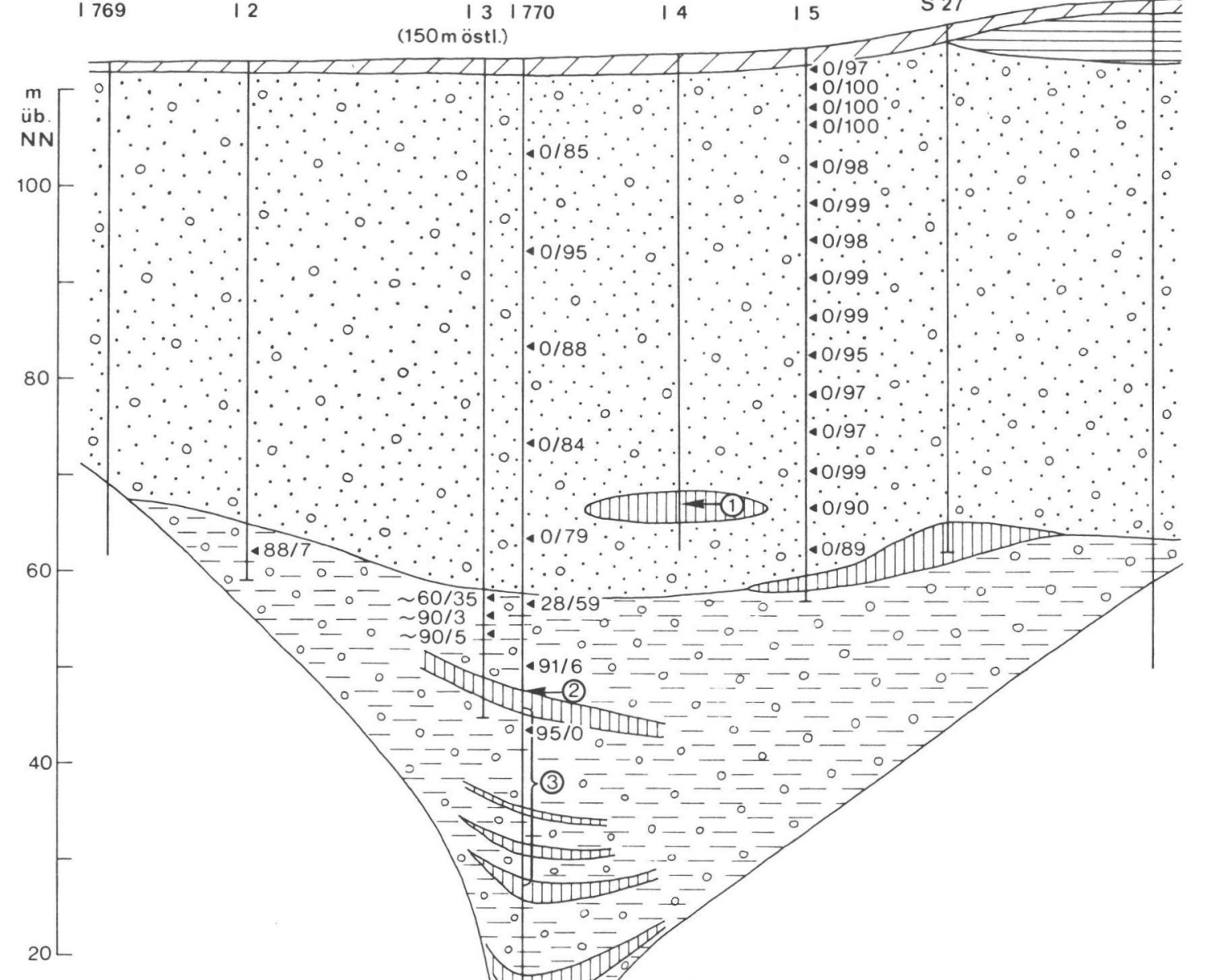

Weichsel bis Holozän
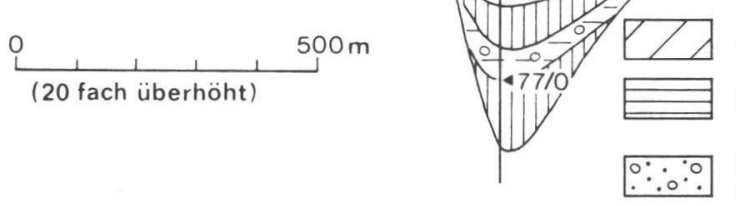

Lößlehm

Edesheimer Ton

Kies, sandig, mit Sand- und

Schlufflagen (Mittelterrasse)

$\begin{array}{ll} & -00 \\ & \text { Sand bis Schluff, kiesig } \\ \text { (Oberterrasse) }\end{array}$

Schluff, tonig, humos,

z.T. Holz und Schnecken führend

- Geröllanalyse

\section{Spät-Drenthe}

ausgehendes Holstein und Drenthe

Altpleistozän bis Holstein

Abb. 7: Mittel- und Oberterrassensedimente bei Edesheim und Hohnstedt. - Geröllanalysen (Fraktion 6,3-12,5 mm): 0/95 bedeutet $0 \%$ Muschelkalk-, $95 \%$ paläozoische Gerölle; die restlichen $5 \%$ sind Buntsandstein und untergeordnet Keuper und Jurageoden. Pollenanalysen (K.-J. MEYER, NLfB Hannover): 1) Holstein-Interglazial oder älter (Proben P 26800-26802), 2) Holstein-Interglazial, Zone VI nach MeYER 1974 (P 33510), 3) vermutlich Holstein-Interglazial, Zone V, und/oder älter (P 33513 - 33523). Lage des Schnitts = A in Abb. 9. 
le des Kiessandes, überwiegend Muschelkalk, daneben Buntsandstein und vereinzelt Kieselschiefer, sind oft gut gerundet, was auf fluviatilen Transport schließen läßt. Eingeschaltet sind Lagen mit eckigen oder nur kantengerundeten Steinen, offenbar Fließerden, die vom benachbarten Hang des StöhneBerges in den Flußkies einspeisen.

$\mathrm{D}$ ist das Profil Ahlshausen 2 (Kap. 2.2). Bei E wurde in zwei Bohrungen für die Auetalbrücke in 36 bzw. $48 \mathrm{~m}$ Tiefe eine $1 \mathrm{~m}$ mächtige Rhume-Kieslage angetroffen (GK 25 Blatt 4125 Einbeck: Abb. 4). In drei Geröllanalysen lag der paläozoische Anteil zwischen 36 und $56 \%$, Muschelkalk war mit $15-38 \%$ beteiligt (Tab. 3, Nr. 7).

Abseits der Bahnstrecke wurden Rhumekies-Vorkommen bei der geologischen Kartierung angetroffen:

In drei Bohrungen im Schlepptal (Tab. 3, Nr. 4, 5) und südlich Ahlshausen, in 10 bis $30 \mathrm{~m}$ Tiefe eingeschaltet in feinkörnige, z. T. humose Beckenablagerungen und Fließerden,

am Wambach-Ufer südlich Olxhausen, 10-18 m über der Leinetalaue, und damit wohl zu hoch für Mittelterrassenkies und im Dünetal.

Im Dünetal konnte die Schichtfolge anhand einer Reihe tiefer Bohrungen untersucht werden (Abb. 8). Ein 10 bis $20 \mathrm{~m}$ mächtiger oberer Abschnitt zeichnet sich durch relativ hohen Kiesanteil aus. Allerdings ist der Schluff- und Tongehalt ebenfalls beträchtlich, wie das Profil an der Bundesstraße 248 nordöstlich Wiebrechtshausen zeigt:

\section{bis $1,5 \mathrm{~m}$ Lößdecke}

- $\quad 2,7 \mathrm{~m}$ Kies, stark sandig, schluffig, tonig; braun (bei 1,8 m die Geröllanalyse Nr. 3, Tab. 3)

- $\quad 3,9 \mathrm{~m}$ Schluff bis Ton, sandig, feinkiesig, mit Tonbändern; rötlichbraun

4,1 m Schluff bis Feinsand, tonig; hellrötlich braun

Unter dem kiesreichen Abschnitt folgen bis $70 \mathrm{~m}$ mächtige feinkörnige Beckenablagerungen, oft feingebändert, z. T. mit Molluskenschill, stark humos und mit Holzresten - mindestens teilweise also warmzeitliche limnische Bildungen. Eingeschaltet sind kiesige Lagen, die sich im einzelnen nicht konnektieren ließen. Die Sedimentverteilung (Abb. 8) scheint ungleichmäßige Absenkung des Dünetals widerzuspiegeln, etwa in Form verschieden alter Teilbecken. Der Grund ist in Subrosion, d. h. Salzablaugung in der Tiefe zu suchen; das Dünetal ist nämlich im Langenholtenser Graben angelegt, einer Fortsetzung des schmalen, Nord-Süd streichenden Juragrabens bei Northeim, auf dessen Randstörung nachweislich Zechsteinsalz aufgedrungen ist (GK 25 Blatt 4325 Nörten-Hardenberg: 72, 73).

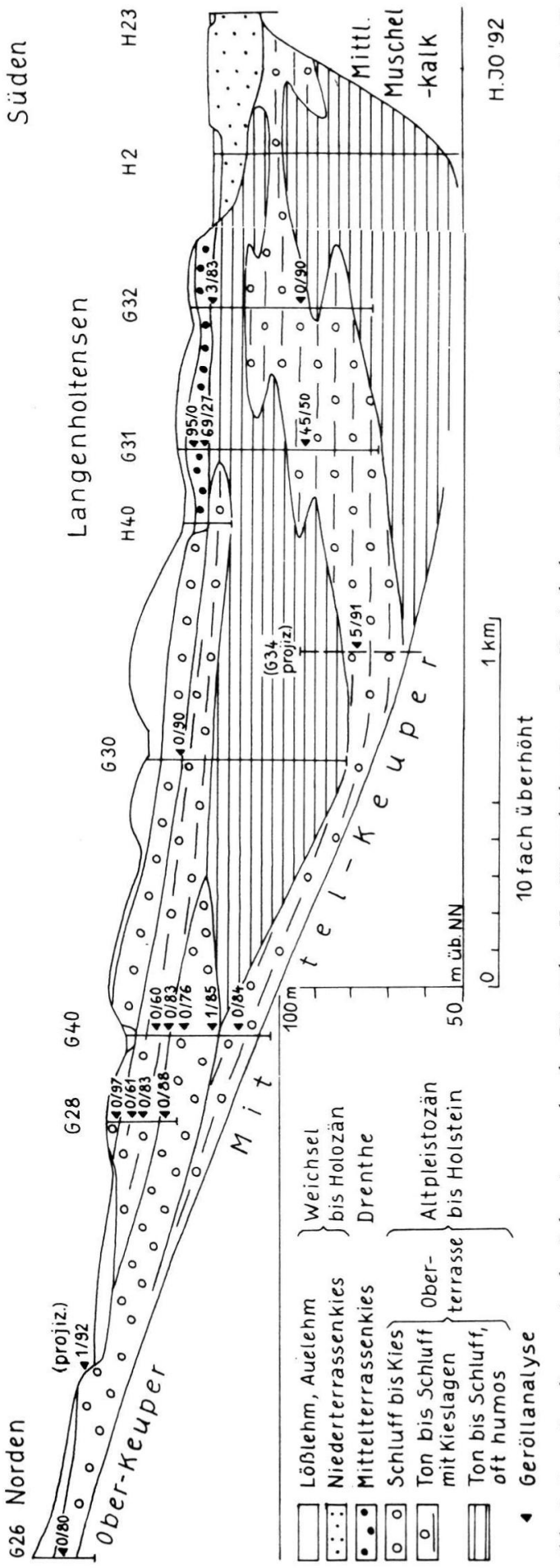


Tabelle 3: Oberterrassenkies bis -schluff der Rhume, Geröllzusammensetzung der Fraktion 6,3-12,5 mm, Nr. 6 12,5-20 mm. ${ }^{1}$ ) untergeordnet auch Malmkalkstein.

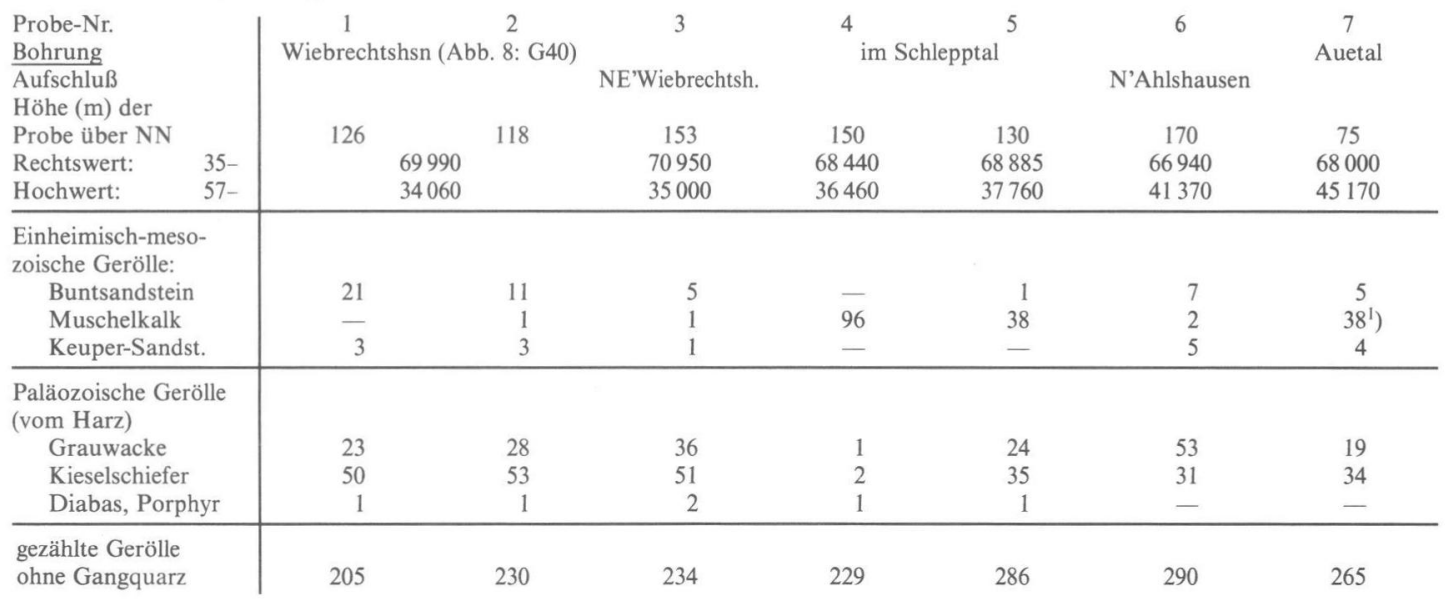

\subsection{Geröllanalyse}

Im Dünetal wurden von verschiedenen Lokalitäten und Teufen 20 Kiesproben auf die Geröllzusammensetzung der Fraktion 6,3-12,5 $\mathrm{mm}$ hin untersucht (Abb. 8), weitere Analysen sind bei LüTTIG (1954: 598) publiziert.

Die Verteilung der nichtlöslichen Gesteine, im wesentlichen Buntsandstein sowie Paläozoikum aus dem Harz, erwies sich als recht einheitlich. Der Buntsandstein liegt, abgesehen von wenigen Ausreißern, zwischen 5 und $20 \%$, Keupersandstein bei $1 \%$ oder wenige Prozent, der Rest sind paläozoische Gerölle (Tab. 3, Nr. 1 - 3, 6).

Muschelkalk ist meistens gar nicht bzw. mit ganz wenigen und stark zersetzten Geröllen beteiligt - in zwei Analysen dagegen mit 5 bzw. $45 \%$. In der Größenordnung dürfte er ursprünglich vorhanden gewesen sein - infolge Solifluktion von den Muschelkalk-Hängen des Dünetales herab, ist dann aber zum größten Teil chemisch gelöst worden. Auch die Grauwacke ist verwittert, in oberflächennahen Proben regelrecht aufgeweicht. Ihr Mengenverhältnis zum (verwitterungsresistenten) Kieselschiefer beträgt etwa 1:1 bis nur 1:5. Zum Vergleich: Der Niederterrassenkies der Rhume bei Northeim führt zwei- bis dreimal so viel Grauwacke wie Kieselschiefer.

Nördlich vom Dünetal läßt sich ein starker seitlicher Zustrom von Fließerden im Oberterrassenkies erkennen. So dominiert im Hohnstedter Rhumelauf der Muschelkalk bereits im Schlepptal (Tab. 3, Nr. 4, und weitere Zählungen), und hat im Vorkommen B bei Hohnstedt den paläozoischen Geröllanteil auf unter $1 \%$ zurückgedrängt. Im Ahlshausener Rhumelauf reicherte sich flußabwärts der Buntsandstein an, auf 60 bis $70 \%$ bei Olxhausen gegenüber 5 bis $20 \%$ im Dünetal. Mit dem Fließerden-Zustrom und mit der starken Verwitterung (und Verschlämmung des Lösungsrückstandes) läßt sich auch der hohe Tonund Schluffgehalt erklären, der das Oberterrassensediment gegenüber dem Mittel- und Niederterrassenkies auszeichnet.

\subsection{Folgerungen zur Flußgeschichte}

Anhand der in Kap. 3.1 beschriebenen Kiesvorkommen lassen sich nördlich Imbshausen drei alte Rhumeläufe nachweisen; zwei weitere Teilstücke - eine Flußschlinge bei Ahlshausen und der Überlauf östlich des Aßberg - sind nach der Geländeform anzunehmen (Abb. 9). Die Flußläufe fielen endgültig in der Holstein-Warmzeit trocken, wie das Vorkommen A bei Edesheim zeigt.

Aus dem Vorkommen Ahlshausen 2 ist zu schließen, daß zumindest dieser Rhumelauf sehr lange Zeit bestand. Die oberste Kieslage (A der Abb. 6) mit massenhaft Harzgeröllen könnte elsterzeitlich sein; denn sie liegt wie das Glaziärvorkommen von Ahlshausen 1 unter dem 3. $\mathrm{fB}_{\mathrm{t}}$. Höheres Alter ist aber nicht auszuschließen, da die $\mathrm{fB}_{\mathrm{t}}$-Horizonte zwischen den beiden Profilen aus arbeitstechnischen Gründen nicht durchverfolgt werden konnten. Die paläozoischen Gerölle der tieferen Lößschichten - über drei $\mathrm{fB}_{\mathrm{t}}$-Horizonte hinab bis zum Ahlshausener Beckenton - sind Reste von cromerzeitlichen, vielleicht auch noch älteren Rhume-Ablagerungen (vgl. Kap. 2.3).

Ursache der Flußverlegungen ist die Hebung des Ahlshausener Gewölbes über einem Zechstein-Salzkissen, das in rund $1000 \mathrm{~m}$ Mächtigkeit mit der Bohrung Northeim 1 durchteuft wurde (FABIAN 1957, JORDAN et al. 1986). Seine Ausdehnung entspricht etwa der Buntsandsteinverbreitung in Abb. 9.

Die Hebung dauerte im Jungpleistozän an und läßt sich für diesen Zeitabschnitt (Holstein bis heute) aus der Verstellung der Rhume-Oberterrasse größenord- 


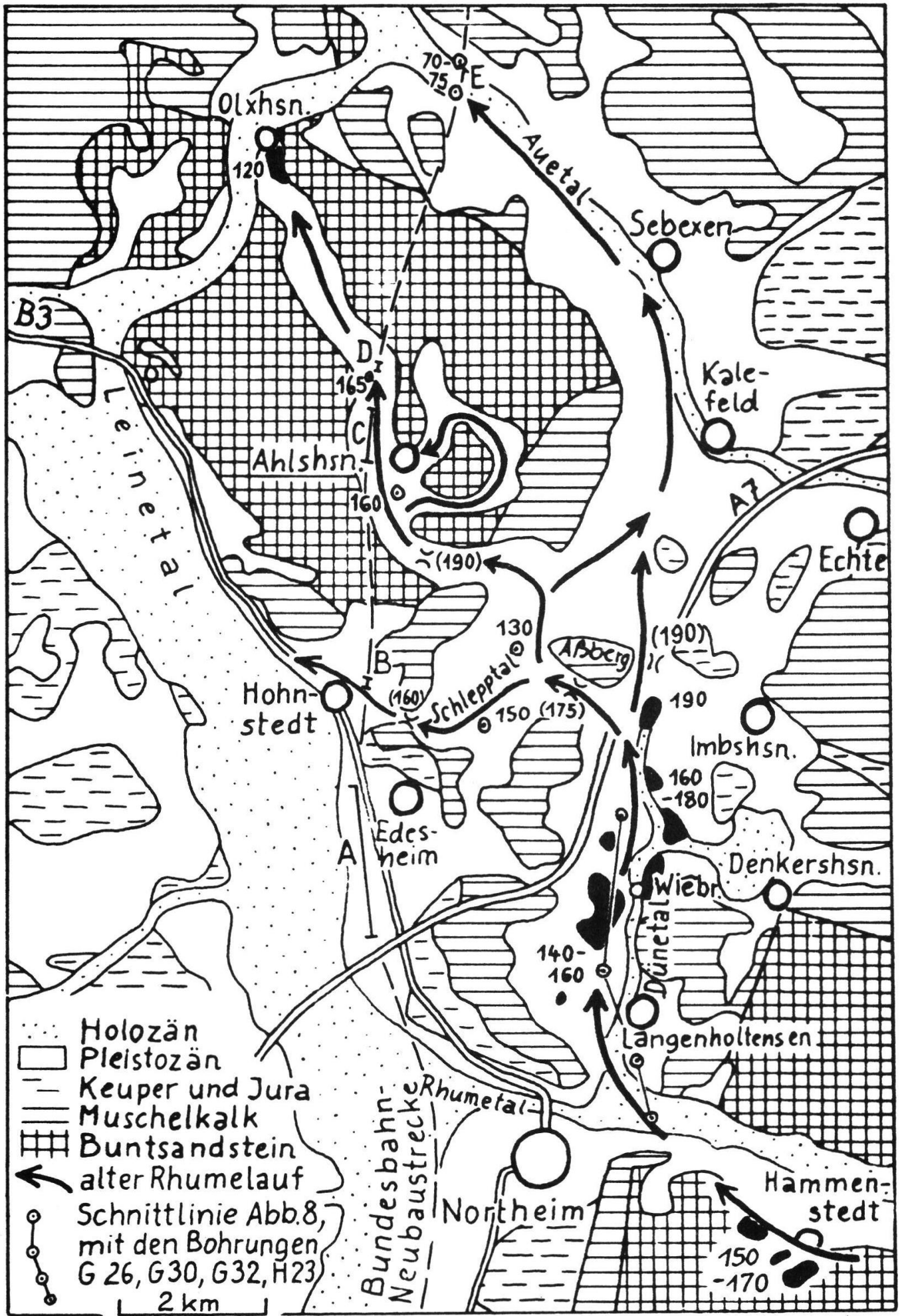

Abb. 9: Vorkommen der Rhume-Oberterrasse an der Oberfläche (schwarz) und in Bohrungen (Kreis mit Punkt), mit Höhenangabe in m üb. NN. In Klammern Höhe der derzeitigen Wasserscheiden abzüglich Quartärdecke. A bis E siehe Tab. 1. 
nungsmäßig abschätzen: Die höchsten Talabschnitte bei Ahlshausen und am Aßberg liegen heute $190 \mathrm{~m}$ über NN - gegenüber 150 m Höhe der OberterrassenBasis bei Hammenstedt, das außerhalb der salztektonisch gestörten Gebiete liegt und sich daher als Bezugspunkt eignet. Veranschlagt man noch ein ursprüngliches Gefälle von $10 \mathrm{~m}$ für die Flußstrecke Hammenstedt-Ahlshausen, so kommt man auf einen Hebungsbetrag von rund $50 \mathrm{~m}$. Setzt man mit ZAGWIJN (1989: Fig. 20) für die Holstein-Warmzeit ein Alter von 350000 Jahren an, so ergibt sich eine mittlere Hebungsrate von 1 bis $2 \mathrm{~cm}$ im Jahrhundert.

Natürlich ist auch im älteren Pleistozän, vor der Holstein-Warmzeit, mit einem Anschwellen des Salzkissens zu rechnen. Offenbar konnte aber die Alt-Rhume durch fluviatile Erosion und zusätzliche Subrosion im Gewölbescheitel die Hebung ausgleichen und ihren Abfluß nach Norden behaupten. Vielleicht war es der kurze Vorstoß des Elstergletschers, der die Rhume bei Northeim staute und so zum Durchbruch nach Westen zwang.

\section{Schriftenverzeichnis}

Fabian, H.-J. (1957): Die Bohrung "Northeim 1" - Ergebnisse eines regionalgeologisch interessanten Aufschlusses am Leinetalgraben. - N. Jb. Geol. Paläont., Abh., 105: 113-122; Stuttgart,

Fromm, K. (1988): Paläomagnetische Untersuchungen zur Alterseinstufung des Lößvorkommens bei Ahlshausen. - Ber. Arch. NLfB, 102789: 14 S.; Hannover. - (Unveröff.).

Jordan, H., mit Beitr. von Büchner, K., Nielsen, H. \& PlauMANN, S. (1986): Halotektonik am Leinetalgraben nördlich Göttingen. - Geol. Jb., A 92: 66 S.; Hannover.
Koenen, A. von (1894): Über die Dislocationen westlich und südwestlich vom Harz und über deren Zusammenhang mit denen des Harzes. - Jb. preuß. geol. L.-Anst., 14: 68-82; Berlin.

LÜTTıG, G. (1954): Pleistozän-Tektonik nördlich Northeim. Geol. Jb., 68: 587-614; Hannover.

- (1960): Neue Ergebnisse quartärgeologischer Forschung im Raume Alfeld-Hameln-Elze. - Geol. Jb., 77: 337-390; Hannover.

Rohdenburg, H. \& Meyer, B. (1966): Zur Feinstratigraphie und Paläopedologie des Jungpleistozäns nach Untersuchungen an südniedersächsischen und nordhessischen Lößprofilen. - Göttinger bodenkdl. Ber., 2: 1-135; Göttingen.

Stremme, H. E., Zöller, L. \& Krause, W. (1991): Bodenstratigraphie und Thermolumnineszens-Datierungen für das Mittel- und Jungpleistozän des Alpenvorlandes. Sonderveröff. Geol. Inst. d. Univ. Köln, 82 (Festschr. Karl Brunnacker): 301-315; Köln.

Zagwijn, W. (1989): The Netherlands during the Tertiary and the Quaternary: a case history of coastal lowland evolution. - Geol. en Mijnbouw, 68: 107-120; Dordrecht.

Geologische Karte von Niedersachsen 1:25 000: Blatt 4023 Eschershausen, Erl.: 189 S.; Hannover 1975. Blatt 4025 Freden, Erl.: 168 S.; Hannover 1984.

Blatt 4125 Einbeck, Erl.: S.; Hannover (im Druck).

Blatt 4127 Seesen, Erl.: 161 S.; Hannover 1976.

Blatt 4225 Northeim West, Erl.: 144 S.; Hannover 1986.

Blatt 4226 Northeim Ost, Erl.: Hannover

(in Druckvorbereitung).

Blatt 4325 Nörten-Hardenberg, Erl.: 148 S.; Hannover 1984.

Manuskript eingegangen am 12. Januar 1993 